\title{
ENHANCING THE FUNCTIONALITY OF THE GROUPS OF AUTONOMOUS UNDERWATER ROBOTS
}

\author{
Valentin E. Pryanichnikov, Vadim V. Chernyshev, Vladimir V. Arykantsev, \\ Alexander A. Aryskin, Stanislav R. Eprikov, Alexander Ya. Ksenzenko, \\ Maxim S. Petrakov
}
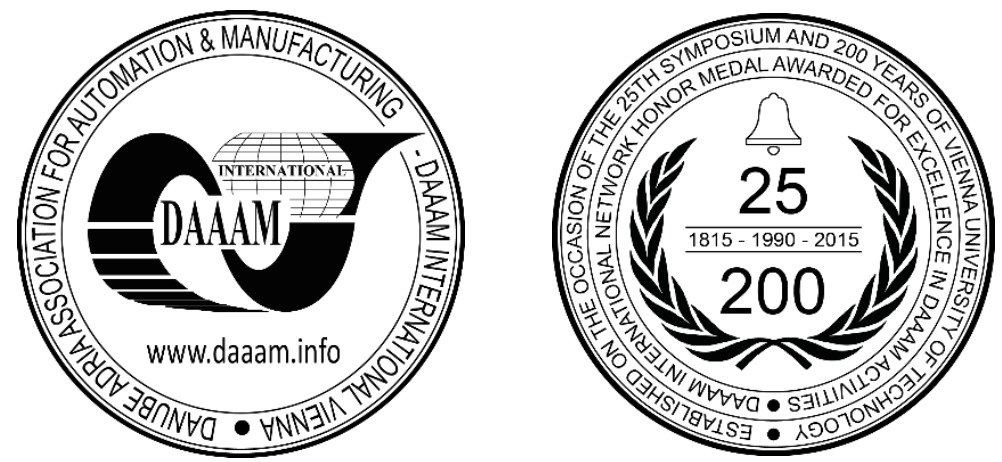

This Publication has to be referred as: Pryanichnikov, V[alentin] E.; Chernyshev, V[adim]; Arykantsev, V[ladimir]; Aryskin, A[leksander] A.; Eprikov, S[tanislav]; Ksenzenko, A[lexander] \& Petrakov M[axim] S. (2018). Enhancing the Functionality of the Groups of Autonomous Underwater Robots, Proceedings of the 29th DAAAM International Symposium, pp.1319-1325, B. Katalinic (Ed.), Published by DAAAM International, ISBN 978-3-902734-20-4, ISSN 1726-9679, Vienna, Austria

DOI: $10.2507 / 29$ th.daaam.proceedings.190

\begin{abstract}
Increasing the efficiency of underwater operations with the help of automatic machines can be achieved by using a robotic complex in a special configuration - with a functional division into three interacting parts: a remote control panel with an operator; a basic underwater walking platform, connected by a cable with the control panel for information and energy supply; a group of autonomous robots with increased maneuverability, having information communication lines with the base station via a contactless channel. Within the framework of this concept, the paper considers and solves the main problems of creating information-control systems for such robotic systems, including the necessary communication tools. One of the key solved problems was constructing the proper sensor-control real-time software system. The paper was partially supported by RFBR, projects 16-07-00935, 16-07-01264, 17-37-50100, 16-07-0081, 16-08-01109.
\end{abstract}

Keywords: service and underwater robots; contactless information and control systems of mobile robots

\section{Introduction}

One of the ways to improve the efficiency of underwater operations in extreme conditions with the help of machines can be the use of robotic complex with functional division into three interacting parts [1]:

- Central control panel with operator in relatively comfortable conditions on the shore or on the accompanying watercraft;

- base station with the ability to move (by its own engines) directly to the area of work and connected with the central control cable (for information and energy exchange);

- group of fully autonomous modules, that directly perform the required work, have increased maneuverability, have information communications with the base station through the ultrasonic channel. 
The organization of the robotic complex with such functional separation also involves the use of a system to ensure the replenishment of the energy reserve of on-board batteries of autonomous modules directly in the working area without surfacing in a non-contact way [2].

However, the principal limitation inherent in such hardware construction of the robotic system is a well-known feature of the ultrasonic communication channel used between each autonomous module and the base station. On the one hand, the desire to increase the maximum possible distance of autonomous modules from the base station involves moving the operating spectrum of the ultrasonic communication channel to a lower frequency interval. That shift in the spectrum will results decrease in the working width of the spectrum, which affects the quality of the television images and, ultimately, the possibility to have a propper operational control by the operator via the central console autonomous module. Thus, at a carrier frequency of about $300 \mathrm{KHz}$, the distance of the autonomous module can be several tens of meters with a satisfactory image quality for operational control of 2-3 frames per second, even when using the compression of the spectrum of the television signal based on its statistical redundancy.

This contradiction is applied to the group of mobile modules may be resolved by using the relay mode coding of the information and control signals between each module of the entire group. To ensure mutual noise immunity, it is necessary to provide a separation of the operating spectra of each of the autonomous modules, that make up the chain of repeaters. At the same time, it is necessary to further narrow the spectrum of the frequency band used by each module at least twice. But for that, it becomes fundamentally possible to increase the maximum distance of remote control for autonomous modules, which significantly expands the functionality of the robotic system.

In particular, it becomes possible in extreme conditions to quickly rescue the autonomous module, which was in an emergency situation, for example, entangled in fishing nets, in the labyrinths of the sunken ship, or in the case of an unacceptable discharge of the onboard battery. Similar problems arise in the development of service robots operating in premises, using ultrasonic and optical information communication channels at distances up to several meters in the absence of line of sight and the corresponding need to install repeaters [3-7].

\section{Design features of data and energy transmission device}

The contactless power supply system is designed for a rapid automatic or semi-automatic replenishment of the electric power supply of mobile robotic devices without the use of direct connection with electrical contacts for the options of ground, underwater, air and space environments.

For robotic systems for ground operations, the primary area of application is the solution of problems in extreme conditions, where the presence of human action is difficult, ineffective or impossible, for example, the performance of works in a high degree of dust, dirt, spray, fog, fuel, aggressive, poisonous, explosive environment. This requires high efficiency and mobility of the robots, which are possible with full autonomy of the robot, as well as the maximum duration of the robot without technological stops.

For robotic systems of underwater operations, the primary area of application is the solution of problems in the conditions of performing work in the deep aquatic environment, as well as in liquid aggressive, combustible environments. Performance of some works by the person is impossible because of unavailability to penetration to the place of performance of works (labyrinths, complicated equipment of the sunken swimming facilities).

The expediency of using this system as part of underwater robotic systems is due to the need for long-term deep work without lifting to the surface for recharging or changing the on-board batteries (lifting depressurization, recharging or changing the battery, subsequent sealing, immersion, search and finding the working area of the work. The effectiveness of underwater operations, provided that there is no need for periodic lifting increases significantly with increasing depth of work.

For robotic systems of air operation, the predominant area of application of this system in the composition of air robotic systems is due to the need for periodic return of the unmanned aerial vehicle to the base for recharging the onboard batteries. At the organization of the intermediate mobile base stations near the operation area of unmanned aircraft eliminates the need for his return to the central database. At the same time, the unmanned vehicle automatically lands on the docking platform of the intermediate base station and promptly performs contactless recharging of its on-board battery.

For robotic systems in different operation space, the use of such method of energy transfer may be very useful. In the operation of autonomous robots in the airless space to replenish the supply of electricity is highly undesirable to carry out this operation with the use of locking. At the same time, there is an effect of welding contacts in vacuum, which limits the possibility of recharging the on-board battery of the autonomous robot by connecting via an external connector. Under such conditions, the contactless power supply system can solve this technical problem.

\section{Principle of operation}

The operation of the contactless power supply system is based on the principle of electrodynamic induction. The system consists of two mutually connected parts: the emitting device and the receiving device. The radiating device includes: a converter of electrical energy, usually DC, into a powerful electrical alternating voltage of high frequency and a converter of this voltage into an electromagnetic field emitted in the desired direction. The radiating device is installed, as a rule, on a stationary or sedentary part of the robotic complex and has a stable power supply. 
The receiving device consists of a receiving antenna, that converts the received electromagnetic oscillations into an electric current, which is then converted into a normalized constant current with parameters suitable for the normal charging mode of the on-board battery of an autonomous robotic device. The receiving device is installed on a fully autonomous robot, contactless power supply of which must be provided.

\section{Technical parameters of the realized devices}

\begin{tabular}{|l|r|l|r|}
\hline \multicolumn{2}{|c|}{$\begin{array}{c}\text { Systems of contactless power supply of underwater } \\
\text { autonomous robot }\end{array}$} & \multicolumn{2}{c|}{$\begin{array}{c}\text { Systems of contactless power supply of ground } \\
\text { autonomous robots }\end{array}$} \\
\hline Range of the action & $0-20 \mathrm{~mm}$ & Range of the action & $10-30 \mathrm{~mm}$ \\
\hline Receiving power & $20 \mathrm{Wt}$ & Receiving power & $300 \mathrm{Wt}$ \\
\hline The efficiency of the system & $25-45 \%$ & The efficiency of the system & $+35 \%$ \\
\hline $\begin{array}{l}\text { Power supply voltage of the transmitting } \\
\text { device }\end{array}$ & $+48 \mathrm{~B}$ & $\begin{array}{l}\text { Power supply voltage of the transmitting } \\
\text { device }\end{array}$ & $+48 \mathrm{~B}$ \\
\hline Receiver weight. & $450 \mathrm{~g}$ & Receiver weight. & $1800 \mathrm{~g}$ \\
\hline
\end{tabular}

\section{Construction}

The radiating device of the underwater robotic complex is mounted inside the body of the mobile base station, which has a cable connection to the central control panel, located on the surface craft or shore, to transmit a television image of telematics information, receive control commands and supply voltage.

The radiating side of the antenna is sealed and made of radio-transparent material. The emitting coil of the transmitting device with the diameter of $55 \mathrm{~mm}$ is placed into a ferrite core forming a radiation pattern oriented by its maximum towards the docking elements. The emitter generator is located in close proximity to the emitter coil and is housed into a metal shield housing to minimize the effect of the emitter coil's powerful electromagnetic field on the sensitive electronic circuits mounted inside the base station.

The receiving device of the underwater robotic system is mounted inside the body of a fully mobile robot, that is not connected by any cables to the base station and the central control panel. Its receiving coil with a diameter of $55 \mathrm{~mm}$ is installed in a ferrite core to efficiently converted the received energy, and placed in a sealed compartment made of radiotransparent material. The board (with the electronic circuit of stabilization) is providing the required parameters of the charging current and protection. It is installed in a metal screen to ensure electromagnetic compatibility of electronic circuits of all systems installed inside the mobile module of the underwater robotic complex. Mutual positioning and holding in the optimal position for efficient power transmission of the transmitting and receiving antennas is provided by the docking device.

The appearance of the transmitting and receiving parts of the contactless power supply system of the underwater robotic complex is shown in Fig.3. The design of the radiating and receiving device ground robotic complex is made similarly, but for greater transmission capacity have radiant the receiving coil with a diameter of $120 \mathrm{~mm}$.

Working of electronic circuits followed by forced cooling. fans the positioning device operates from television and optical sensors.

The mode of the most effective from the point of view of the efficiency of non-contact power transmission for the underwater version of the robotic system is provided both in fresh and salt sea water, maintaining the distance between the transmitting and receiving coils of the order of $10 \mathrm{~mm}$. For the land version, this interval is at a distance of $16 \mathrm{~mm}$.

\section{The principle of operation of the hardware and software platform}

The first iteration of the hardware-software platform development was used to control the underwater walking machine MAK-1 (Fig. (1) [8]. Tests of the device on the White sea and water bodies of the Volga-Akhtuba floodplain [911] showed the need for a significant modernization of its control system. The functional diagram of the proposed hardware-software platform is shown in Fig. 2.

The scheme can be divided into two main functional blocks: yellow block (left) - the control panel of the underwater robot MAK-1, which can be made as a shockproof case with a laptop, joystick and USB-CAN adapter. This panel would be in the operator's boat or on the shore. Frequency converters would be located directly on-board the underwater vehicle, in a sealed housing. It would also have a board for receiving commands from the control panel and generating control signals for frequency converters (serial interface of the state of emergency). Since the logic voltage levels of the PE and Arduino interface are different, the control signal would be amplified through the relay unit, which would provide galvanic isolation to improve reliability and prevent the main Board failure in case of voltage surges on high-voltage lines. Also Arduino mPC would collect data from on-board sensors. 


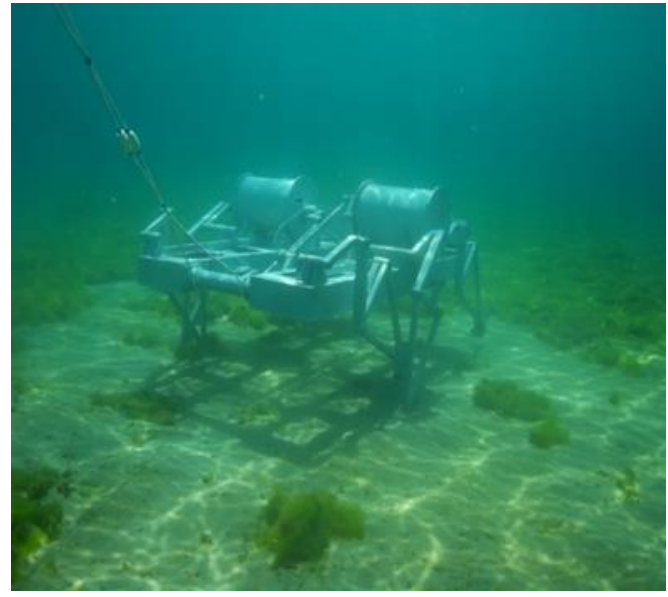

Fig. 1. Underwater walking machine MAK-1

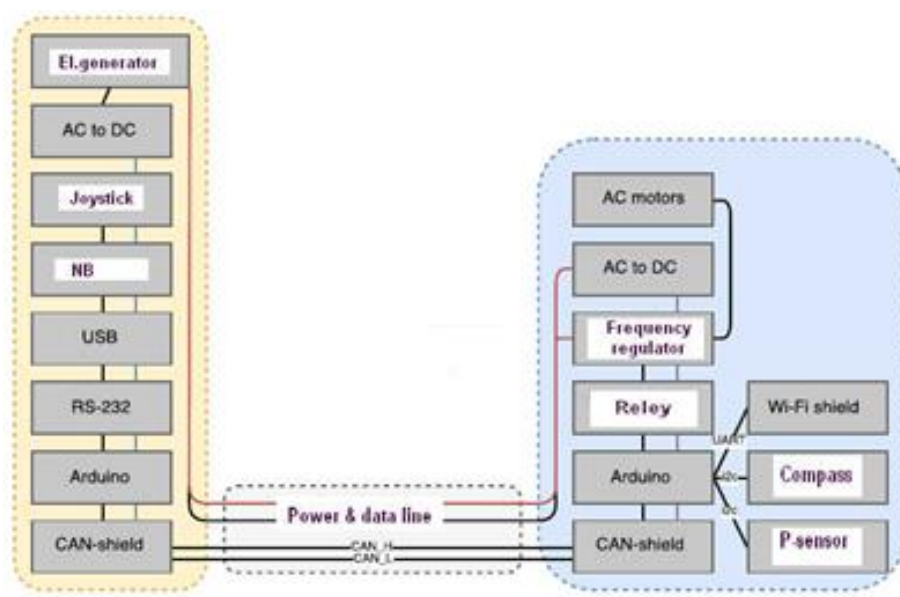

Fig. 2. Functional diagram of the basic platform.

Various options for the implementation of communication between the control panel and the on-board system were considered. The most popular option with Ethernet did not fit because of its short distance of operation. The maximum allowable cable length without repeaters is only 100m, and the channel speed may be excessive for the tasks.

On the other hand, a fiber optic cable could be used instead of a twisted pair cable. It is very well used in the underwater robot Nereus (one of the first deep-sea robots for $10 \mathrm{~km}$ ) Institute Woods Hole Oceanographic Institute (Institute specializing in deep-sea vehicles). The main limit of the cable (length $10 \mathrm{~km}$ ) was due to the limiting high pressure at these depths. Potentially, the fiber optic cable can extend even longer distances $(16 \mathrm{~km})$ without a repeater, with good quality transmitting video stream.

But this option is not suitable because of the difficulties of working with fiber optic cable, namely in the process of winding and unwinding the cable when the machine is immersed in depth. In case of improper use, if the small kinks in the deformation of the ruptures of the fibers in the cable becomes operational. This problem in the Nereus robot was solved by disconnecting the cable when the robot floated to the surface in automatic mode and the cable was lifted separately by the robot (the speed of robot ascent is much higher than the speed of cable winding). In this case, this method is not suitable because the manual control of the robot is lost when the cable is disconnected.

Also considered were the interfaces that can be integrated with the Arduino mPC, namely: i2c, spi, rs-232. According to the above requirements (working distance), the most suitable was the industrial network standard - CAN. Under this Protocol there are ready-made boards-drivers software packages to work with it.

Its maximum distance of operation without rehearsals is 5000 meters at a speed of $10 \mathrm{kbit} / \mathrm{s}$, which is sufficient to control the motors and collect data from the onboard sensors. On the side of the control panel can be used a pair of Arduino+CAN or CAN-USB adapter. The first option was chosen to be independent of the adapter manufacturer's drivers. The information cable, in turn, is protected by the screen from external interference, including from the side of the power cable. The screen is grounded to the underwater vehicle body.

Despite all the advantages of this scheme, the following disadvantages were identified:

- $\quad$ More components of the system - less reliability. In case of failure of the Arduino or any software error lost control of the underwater vehicle, which is particularly critical if the robot is already at great depth.

- $\quad$ More complex implementation due to the need to produce a hardware on-board system, as well as the manufacture of a sealed enclosure.

Given the above drawbacks, the scheme has been redesigned into a simpler one (Fig.2), which excluded any equipment on-board the robot, including frequency converters. Control of the engines was carried out directly on the power cables, that came from the control panel, where the frequency converters, the operator's computer, and the joystick were located. The interaction of PC and PE was replaced with a parallel interface, which meant some kind of adapter in the form of pairs of Arduino + relay controller on the serial interface of the PE, which runs on the RS485 Protocol.

For Fig.3-5 shows one of the variants of the implemented software for manual and program control of the underwater robot MAC-1. 


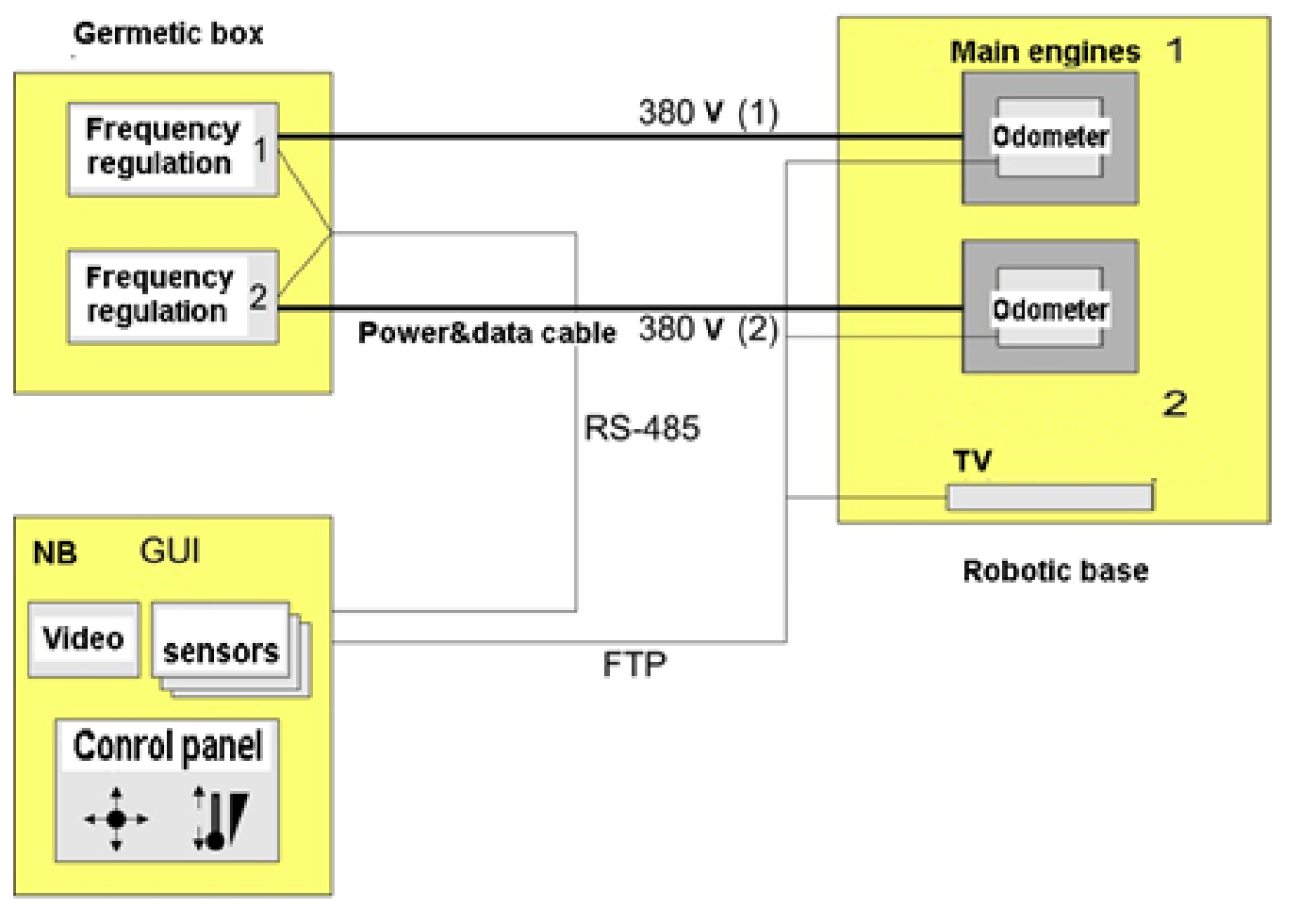

Fig. 3. Revised functional diagram of the operation of the platform

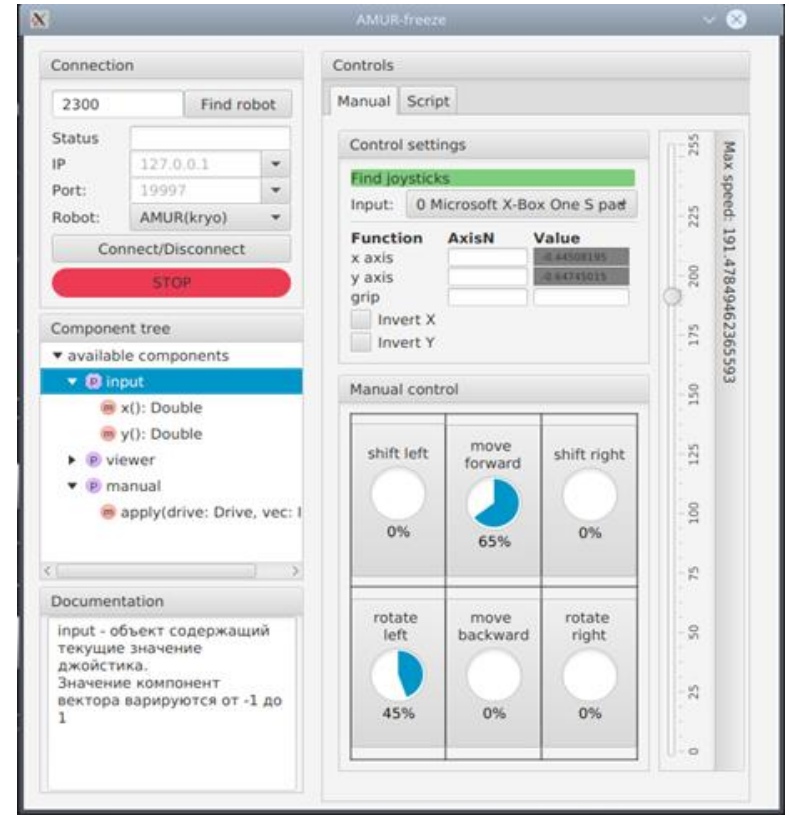

Fig. 4. AMUR-freeze graphical interface in manual mode.

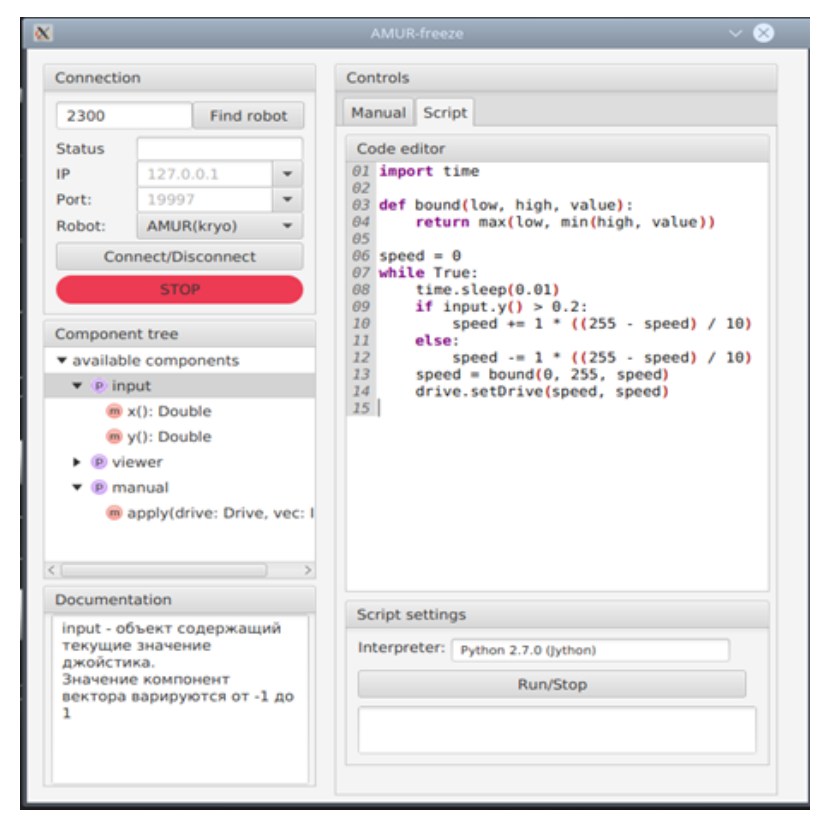

Fig. 5. GUI AMUR-freeze mode control software.

For the correct operation of the developed software system AMUR-freeze on the operator's computer it was necessary to install JVM 1.8, which is supplied. AMUR-freeze was implemented using the Scala programming language, and JavaFX was chosen as a software package for the graphical interface. Control can be carried out at the expense of one of the input devices: mouse, keyboard or game controller-gamepad (any gamepads, that support DirectInput or XInput driver). Was tested gamepads such as the Xbox One controller, Logitech F310 Gamepad Defender Cobra M5 USB.

The main difference from the existing solutions is the ability to programmatically adjust the program during its execution (without recompiling the project) or writing relatively small (about 200-300 lines) scripts. Example of use: suppose you want to make a smooth start of the robot movement, but when you press the keyboard button, the robot is driven at full power because the keyboard key can be either pressed or clamped. 
For such a case, it is possible to write a script that would intercept the values from the joystick, transform the values according to a certain law (linear for example) and pass them to the movers. It is also possible to intercept values not only from input devices, but from sensors installed on the robot It is also worth noting that the functionality of this program is of a general nature and is successfully used to control ground mobile robots such as AMUR (International laboratory "Sensorika") and Robotino (FESTO). The graphical interface is divided into several visually separated sections, which are described below.

Connection: In this section, you configure the Internet connections for the robot. You must specify the port and IPaddress of the program running on the robot-driver. In the case of the MAC-1, only the port of the RS-485 adapter is connected to the operator's computer and is indicated. In some cases (depending on the type of the driver program) it is possible to scan robots in the network due to the broadcast signal through the "Find robot" button and the subsequent auto-complete of the "port" and "IP-address"fields. The "Robot" field indicates the communication Protocol, which can be specific for the robot.

Component tree: The main purpose is to inform the operator about the available components and their properties for program control. It is a tree-like structure where the top - level nodes are the components themselves and their child nodes are the methods (in terms of OOP) of the components. The method can take arguments of a certain type (specified after the "sign") and return a value. By selecting a component or method, the user gets more detailed information in the "Documentation" section. For example, the picture shows the components to get the value of the input device ("Input") and the component of the robot mover.

Documentation: Description of operation and properties of the selected component in the "Component tree" section.

Control settings : In this section, the input device for manual control of the robot is selected. Since the program axis number of the joystick handle varies depending on the type of joystick, it is possible to select the axis numbers or invert the axes (or with different switching of power cables attached to the motors). If the joystick is not connected, it is controlled by six keyboard keys (WASDQR) or by buttons in the program window.

Manual control: Buttons for manual control of the mouse in the absence of a joystick or keyboard. Also there is an indication that reflects the level of the handle in the most extreme position.

Script control: In this section, the source is edited when the script is in the "Code editor" field. As the programming language in which the script is written, Python was chosen for its ease learning and use. Since AMUR-freeze is implemented on JVM (Java virtual machine) for easier integration, the implementation of Python for JVM (Jython) was chosen, which is already built into AMUR-freeze. As a result, the user does not need to download/install additional sortware. At the bottom of the interface, the script is started and stopped, as well as the field for standard I/O.

AMUR-freeze (web): In addition to the AMUR-freeze implemented on the basis of JVM, its web browser version was implemented, which would not require any pre-installed software, except for a web browser. The functionality is almost identical to the AMUR-freeze plus there is the ability to work in multi-user mode.

\section{Conclusion}

The results of the work may be installed on demand in the developing walking robotic systems, designed for underwater engineering and for any new industrial technologies for the development of seabed resources.

\section{Acknowledgments}

This paper was partially supported by the Russian Foundation for Basic Research, Project No. projects 16-07-00935a, 16-07-01264a, 17-37-50100, 16-07-0081a, 16-08-01109a.

\section{References}

[1] Bielich T., Ksenzenko A.Ya., Kirsanov K.B., Pryanichnikov V.E. Increasing uptime underwater inspection robotic system // Information-measuring and control system, Radiotechnika. 2015. V. 13. № 7. P. 51-56, ISSN: $2070-08147$.

[2] B. Katalinic, A.Ya. Ksenzenko, S.V. Kuvshinov, Yu.S. Marzanov, E.A. Pryisev, V.E. Pryanichnikov, R.V. Khelemendik, S.R. Epikov. The development of distributed software and hardware for «Robotariums»//EXTREME ROBOTICS. Proceedings of the International Scientific and Technological Conference. - Saint-Petersburg: «AP4Print», 2016, pp.459-460 (480 p.)

[3] Pryanichnikov V.E., Biyelich T., Vitsan D., Katalinich B., Kirsanov K.B., Kuvshinov S.V., Marzanov Y.S., Podurayev Y.V., Khelemendik R.V., Prysev E.A., Ugleshik A., Kharin K.V. Development of education technologies 
and the network of associated laboratories-robotariums. Jornal "Data Measuring and Control Systems", M.: Radiotechnica, 2015, т.13, N7 - ISSN 2070-0814, C.7-25

[4] Pryanichnikov V.E., Khelemendik R.V. About building of a special logical language for the problems of intellectual robotics and the research of large formulas // Computer Science and Information Technology: Materials of International Scientific Conf. - Saratov: The publication Centre "Science", 2018, pp. 425-428.

[5] MAC-1 - underwater walking robot / V. V. Chernyshev, V. V. Arykantsev // Robotics and technical Cybernetics. 2015. No. 2. C. 45-50.

[6] The testing of an underwater walking apparatus in the conditions of water objects of the Volga-Akhtuba floodplain / V. V. Chernyshev, V. V. Arykantsev // System environment monitoring. 2017. № 8 (28). 24-29.

[7] Kirsanov K., Levinsky B., Pryanichnikov V. Integrating software for intelligent robots // Informational-Measuring and Control Systems, M.: Radiotechnika. 2009. V. 7. № 6. P. 35-43.

[8] Pryanichnikov V.E., Aryskin A.A., Eprikov S.R., Kirsanov K.B., Khelemendik R.V., Ksenzenko A.Ya., Prysev E.A., Travushkin A.S. Technology of Multi-Agent Control for Industrial Automation with Logical Processing of Contradictions, Proceedings of the 28th DAAAM International Symposium, 2017, pp.1202-1207, B. Katalinic (Ed.), Published by DAAAM International, ISBN 978-3-902734-11-2, ISSN 1726-9679, Vienna, Austria. DOI: 10.2507/28th.daaam.proceedings.167.

[9] Davydov D.V., Eprikov S.R., Kirsanov K.B., Pryanichnikov V.E. Service Robots Integrating Software and Remote Reprogramming, Proceedings of the 28th DAAAM International Symposium, 2017, pp.1234-1240, B. Katalinic (Ed.), Published by DAAAM International, ISBN 978-3-902734-11-2, ISSN 1726-9679, Vienna, Austria, DOI:10.2507/28th.daaam.proceedings.172. http://daaam.info/Downloads/Pdfs/proceedings/proceedings_2017/172.pdf

[10] Design and underwater tests of subsea walking hexapod MAK-1 / Chernyshev V.V., Arykantsev V.V., Gavrilov A.E., Kalinin Y.V., Sharonov N.G. // Proceedings of the ASME 2016 35th International Conference on Ocean, Offshore and Arctic Engineering OMAE2016 / Ocean, Offshore and Arctic Engineering (OOAE) Division of The American Society of Mechanical Engineers (ASME), Pusan National University. [Busan], 2016. - 9 p.

[11] Subsea tests of walking unit MAK-1 / Chernyshev V.V., Gavrilov A.E., Arykantsev V.V. // Joint International Conference Minerals of the Ocean-8 \& Deep-Sea Minerals and Mining-5: Abstracts / VNII Okeangeologia. Saint Petersburg, 2016. C. 66-70. 